\title{
Wearable and Wireless Distributed Multi-site FES Prototype for Selective Stimulation and Fatigue Reduction: A Case Study
}

\author{
Haipeng $W A N G^{1,2}$, Zhengyang $B^{3}$, Yuxuan $Z H_{O U}^{3,4}$, Fei $L^{1}$, Keping $W A N G^{1}$, \\ Xiao-Ying $L U^{3,5}$, Zhi-Gong WANG ${ }^{1,5}$ \\ ${ }^{1}$ Institute of RF-\& OE-ICs, Southeast University, 210096 Nanjing, China \\ ${ }^{2}$ School of Electronic and Information Engineering, Sanjiang University, 210012, Nanjing, China \\ ${ }^{3}$ State Key Lab of Bioelectronics, Southeast University, 210096 Nanjing, China \\ ${ }^{4}$ Dept. of Biomedical Engineering, Nanjing Medical University, 211166 Nanjing, China \\ ${ }^{5}$ Coinnovation Center of Neuroregeneration, Nantong University, 226001 Nantong, China \\ luxy@seu.edu.cn, zgwang@seu.edu.cn
}

Submitted April 29, 2020 / Accepted October 19, 2020

\begin{abstract}
The objective of the work is to evaluate the feasibility of improving selective stimulation and reducing muscle fatigue in upper extremity rehabilitation with a selfdesigned multi-site functional electrical stimulation (FES) prototype. The design of the prototype is a distributed architecture concept, and all the modules communicate within a single local area network controlled by the Android application (APP). To improve the efficiency of the prototype, the APP was developed to utilize a real-time online algorithm to perform a rapid real-time search for the optimal stimulation site. One healthy subject participated in a multi-site FES trial consisting of a search for the optimal stimulating electrode test and a fatigue stimulation test. Comparing the results of the online automatic search for the optimal stimulation site test with the results of the offline analysis, the average Location Error for the extension and flexion motions were $1.2 \mathrm{~cm}$ and $2.5 \mathrm{~cm}$, respectively. For the fatigue stimulation test, all the assessments of the multi-site sequential stimulation group were higher than those of the conventional stimulation, with a significant 193\% higher Fatigue Index $(P=0.003)$ and 300\% longer Fatigue Time $(P=0.005)$. These results suggest that multi-site FES may exert positive effects on selective stimulation and stimulation fatigue reduction in healthy subjects.
\end{abstract}

\section{Keywords}

Functional electrical stimulation (FES), wireless communication, local area network, wearable device

\section{Introduction}

Functional electrical stimulation (FES) can be considered to serve as a communication bridge between the peripheral nervous system (PNS) and a damaged central nervous system (CNS) through the use of a series of artificially organized electrical pulses applied to the motor units (MUs) of target paretic muscles. With the help of FES, patients can recover various physical movements, such as grasping [1]. Combined with control splints, Long and Masciarelli first used FES to help hemiplegic patients achieve hand grasp rehabilitation [2]. The most notable feature of the first therapeutic FES device is its portability [3].

However, there are three limitations to the application of surface FES: 1) discomfort and pain, 2) insufficient stimulation selectivity, and 3) rapid muscle fatigue [4]. Therefore, the stimulation site needs to be individualized to induce selective movements of the hand and wrist. FES requires a higher frequency than is normal in the human body, and a large number of MUs producing non-coordinated, synchronized activation can result in rapid fatigue.

Since Nathan's first use of surface array electrodes placed at the forearm muscles in one spinal cord injury (SCI) patient with multi-channel stimulation [5], selective stimulation of multiple sites has gained increasing attention. Fujii first designed a selective FES system using surface array electrodes [6]. The multi-pad electrode was developed from surface array electrodes. Thierry Keller designed a $10 \times 6$ array of multi-pad electrodes embedded in clothing textiles for easy wear [7] and used an evaluation system to measure the strength of the flexion of each finger during grasping. The "Actitrode" system employs a 24position circular array electrode using conductive ink and biocompatible textile fibers [8], leading to a more integrated and convenient design. Malešević proposed the use of a multi-pad electrode with a pressure sensor and grasping strength measurement system [4] and an irregularly arranged multi-pad electrode in regard to the size and shape of the electrode site that depended on the on characteristics of the forearm [1]. These improvements increased the lateral coverage of the forearm and improved the selectivity. O'Dwyer et al. presented a multi-pad electrode FES system 
with a self-configuration electrode algorithm that used a wearable glove with feedback sensors for measuring wrist response [9]. Recently, a method for selecting the stimulation sites was proposed by estimating from the differences of recorded EMG activity maps on the nonparetic and paretic forearms [10]. A multi-site electrode system (HandNMES) with six customized wearable electrode arrays and an external stimulator was developed aimed for grasp training and assistance [11]. However, most FES systems with multi-site stimulation require multiple wires and are cumbersome to wear. In addition, stimulation electrode optimization and the searching algorithm have high computational complexity and time requirements.

This paper presents a new wearable multi-site FES prototype designed using a wireless and distributed architecture concept. The wireless control operation can facilitate improved convenience and efficiency. An Android application (APP) that uses a simple on-line algorithm was developed to perform fast, real-time searches for the optimal stimulating electrode, and the optimal electrodes correspond to twelve movements, including wrist extension/flexion and extension/flexion of the five fingers. The purpose of this case study was to evaluate the feasibility of improving selective stimulation and reducing stimulation muscle fatigue in upper extremity rehabilitation with a selfdesigned multi-site FES prototype.

\section{Methods}

\subsection{Participant}

One healthy subject (male; $30 \mathrm{yrs}$ ) participated in this study. The subject was asked to refrain from any strenuous exercise of his upper extremity for at least 24 hours before the start of the study. The subject provided written informed consent before participation, and this study was approved by the Human Subjects Review Board of Southeast University.

\subsection{Instrumentation Design}

\subsubsection{Hardware Description}

Figure 1 shows the intelligent and wearable multi-site FES prototype. The system is designed in a distributed architecture concept consisting of five modules: an Edison wireless stimulator, a wireless electrode driving module, a multi-pad stimulation electrode, an Edison wireless data glove and an intelligent control terminal (smartphone). Wi$\mathrm{Fi}$ is used for wireless communication among the EdisonSTIM stimulator, the wireless electrode driver module, and the wireless data glove and control terminal. In this prototype, a Huawei C8813Q Android mobile phone was selected as the control terminal device. The stimulator and the data glove were developed using an Intel® Edison com pute module (ECM). The ECM wearable platform contains a $500-\mathrm{MHz}$ dual Atom $^{\mathrm{TM}}$ core and a $100-\mathrm{MHz}$ Quark $^{\mathrm{TM}}$

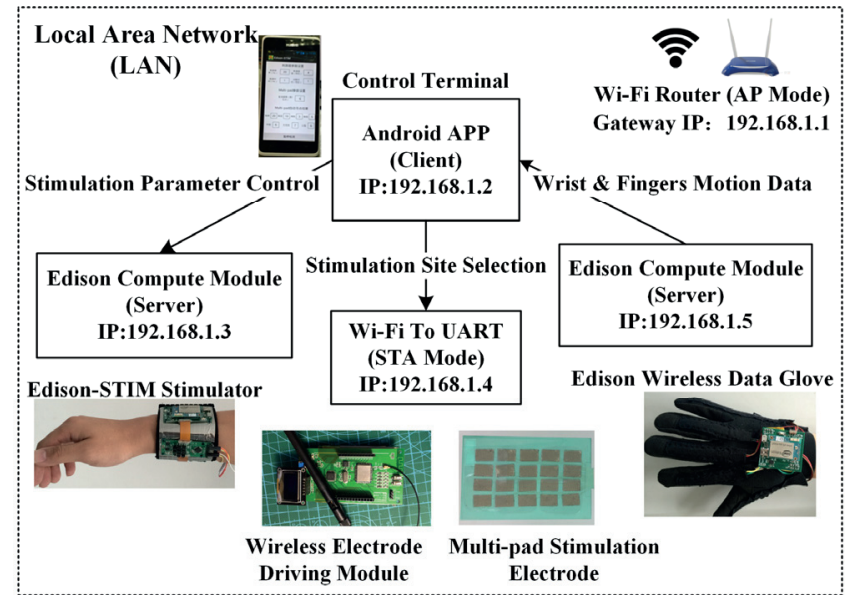

Fig. 1. Representative block diagram and wireless communication network architecture of the proposed wearable multi-site FES prototype.

microcontroller with the Yocto Linux v1.6 operating system, which can perform rapid prototyping of wearable and wireless products. Use of this distributed architecture design, therefore, can facilitate wireless control and increase the convenience of wear and use.

The TCP/IP protocol and "Socket Objects" in the Java language were used to achieve wireless sensor network [12] communication between modules. All the modules worked within the same local area network (LAN) provided by a Wi-Fi router operating in access point (AP) mode. The IP address of the gateway was 192.168.1.1. Since multiple modules communicated within the system, multiple IP addresses were required, and the socket ports and IP addresses needed to be determined in advance when using socket communication. Therefore, in the proposed prototype, all the modules and devices communicated with fixed IP addresses (generally, a fixed IP address can be assigned to the device in the router configuration software). The Android APP in the control terminal was designed as a client socket and was developed to control the stimulator, electrode driving module, and data glove. The APP is responsible for transmitting stimulation parameter control instructions and stimulation site selection commands to the stimulator and the electrode driving module, respectively, and for receiving the motion data of the wrist and fingers from the data glove.

Figure 2 shows the site distribution map, the material structure and a photograph of the designed rectangular $\left(11 \times 15 \mathrm{~cm}^{2}\right)$ array stimulation electrode. The flex printed circuit (FPC) material of Polyimide/Polyester was used as the electrode substrate, and the conductive 20 pads were arranged in a $5 \times 4$ array with the number "\#1" to "\#20" labelled as the site positions in order. Each site had a stimulation size of $2 \mathrm{~cm}$ by $1.5 \mathrm{~cm}$ with the interpad distance of $2.5 \mathrm{~cm}$ axial and $2 \mathrm{~cm}$ transversal (Fig. 2a). The electrode fabrication structure of the material, from the bottom layer to the top layer, was composed of a metal pad, conductive cloth and high-impedance thin-layer hydrogel (Fig. 2c). In addition, the multi-pad electrode was connected to the wireless electrode driving module via a 20-pin connector. 


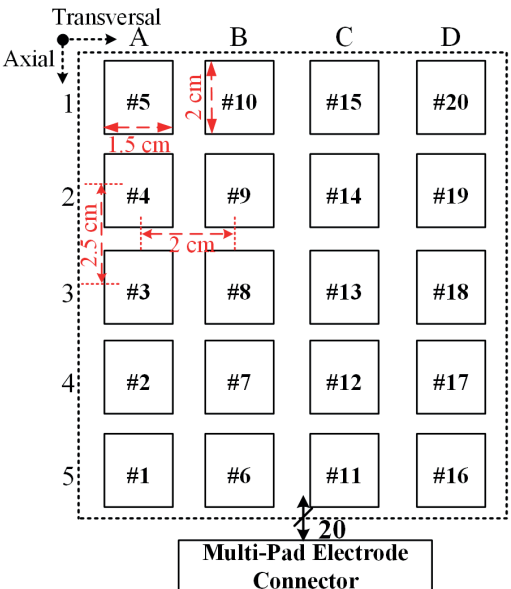

(a)

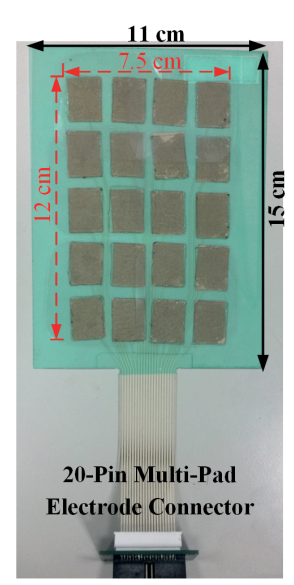

(b)

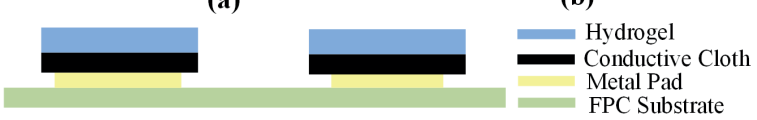

(c)

Fig. 2. (a) Multi-pad electrode site distribution map. (b) Photograph of the designed multi-pad stimulation electrode. (c) Schematic diagram of setup material structure of one site of the multi-pad electrode.

The electrode driving module (illustrated in Fig. 1) consisted of a low-power consumption microcontroller (MCU) ARM $^{\circledR}$ Cortex $^{\circledR}$-M3 (STM32F1038BT6, ST, Inc.), a Wi-Fi module (HLK-M30, Hi-Link Electronic, Inc.), an organic light-emitting diode (OLED) display, a low drop-out regulator circuit and an electrode selection circuit. The HLK-M30 operated in station (STA) mode to serve as the receiving node of the MCU. Considering the weak signal power of the PCB board antenna, this module utilized an external antenna to improve the communication accuracy. The intelligent control terminal sent the channel selection instructions to the wireless electrode driving module through the Wi-Fi module to the MCU, and the OLED displayed the selected channel information. The MCU then generated the corresponding selected electrode gating control signal to the electrode selection circuit, which was composed of 20 channels.

The Edison wireless data glove (illustrated in Fig. 1) is a medium-sized right-handed glove containing eight bend sensors and one motion sensor MPU6050. The configuration of the bend sensors included two 2.2-inch sensors placed over each dorsal side of the proximal interphalangeal (PIP) and metacarpal (MCP) joints in three fingers (index, middle, and ring), and only one 4.5-inch sensor was placed over the MCP joint for the thumb and little finger. To ensure the sensors remained in position, they were fixed with one layer of Lycra fabric. The output data of the bend sensors and the motion sensor are recorded through an Analog-to-Digital Converter (ADC) ADS7971 (Texas Instruments, USA) with a serial peripheral interface (SPI) and an inter-integrated circuit (I2C) bus in ECM, respectively. Therefore, eleven rotations at 10 joints can be measured by the Edison wireless data glove: flexion and extension of the PIP and MCP joints of the index, middle and ring fingers; flexion and extension of the MCP joints of the thumb and little finger; ulnar and radial deviation; ex- tension and flexion of the wrist; and pronation and supination of the forearm. Many researchers have worked to improve the measurement accuracy and stability and the linearity of the resistive bend sensors used for the assessment of finger movements and hand motions [13-15]. Gentner [15] proposed that using a suitable resistor in parallel with bend sensors could efficiently improve the linearity between the output voltage of the sensor conditioning circuit and the bending angle. In our prototype, the method of paralleling the sensor with a resistor was not adopted due to the requirements of a wide range of bending angle detection and a high dynamic range. Therefore, after the linearity test of the bend sensor, the segmented fitting method was used to calibrate the bend sensor system to obtain accurate measurements.

The stimulator circuit was based on the complementary current source and time division multiplexing (CCSTDM) technique as previously described [16]. A pair of Wilson current mirrors (a current source and a current sink) constitutes the complementary current source circuit in the Edison-STIM stimulator. Therefore, under the electrode gating control signal of the electrode driving module, the current source was connected to the multi-pad electrode and the rectangular array electrodes were used as cathodes. An extra gelled stimulation electrode $\left(4 \times 4 \mathrm{~cm}^{2}\right)$ was placed $2 \mathrm{~cm}$ away from the multi-pad electrode near the elbow olecranon. It was directly connected with the current sink of the stimulator and used as the anode.

\subsubsection{Method of Optimal Stimulation Electrode Determination}

The subject was instructed to wear the multi-site prototype and perform twelve target movements with voluntary contraction (voluntary mode, and the stimulator was turned off), during which motion data were collected, including wrist extension, wrist flexion, five-finger extension and flexion. The task was repeated 10 times. The motion data were recorded and saved in the file system of the Edison compute module in the wireless data glove.

1) Off-line criterion applied for the selection of the "optimal electrode": By acquiring the motion data in the stimulation and voluntary modes, the aggregate difference error $\Delta_{\mathrm{i}}(i=1,2,3, \ldots, 20)$ of each pad between stimulation response data and the target motion autonomic response data of polling electrode are defined and modified from reference [17].

$$
\begin{aligned}
& \Delta_{i}=\sqrt{\Delta_{\text {finger }}^{2}+\left(\frac{\Delta_{\text {EXT/FLEX }}}{\varphi_{\text {EXT/FLEX }}}\right)^{2}+\left(\frac{\Delta_{\text {ULNRAD }}}{\varphi_{\mathrm{ULN} / R A D}}\right)^{2}+\left(\frac{\Delta_{\text {PRO/SUP }}}{\varphi_{\text {PRO/SUP }}}\right)^{2}}, \\
& \Delta_{\text {finger }}^{2}= \\
& \frac{1}{8}\left[\begin{array}{l}
\left(\frac{\Delta_{\mathrm{TMCP}}}{\varphi_{\mathrm{TMCP}}}\right)^{2}+\left(\frac{\Delta_{\mathrm{IPIP}}}{\varphi_{\mathrm{IPIP}}}\right)^{2}+\left(\frac{\Delta_{\mathrm{IMCP}}}{\varphi_{\mathrm{IMCP}}}\right)^{2}+\left(\frac{\Delta_{\mathrm{MPIP}}}{\varphi_{\mathrm{MPIP}}}\right)^{2}+ \\
\left(\frac{\Delta_{\mathrm{MMCP}}}{\varphi_{\mathrm{MMCP}}}\right)^{2}+\left(\frac{\Delta_{\mathrm{RPIP}}}{\varphi_{\mathrm{RPIP}}}\right)^{2}+\left(\frac{\Delta_{\mathrm{RMCP}}}{\varphi_{\mathrm{RMCP}}}\right)^{2}+\left(\frac{\Delta_{\mathrm{LMCP}}}{\varphi_{\mathrm{LMCP}}}\right)^{2}
\end{array}\right]
\end{aligned}
$$


Note that $i$ represents the electrode number as illustrated in Fig. 2(a). Other variables in (1), (2) are explained below. The $\Delta_{\mathrm{TMCP}}$ represents the thumb MCP joint angle error (stimulation response and autonomic response), $\varphi_{\mathrm{TMCP}}$ represents the thumb MCP joint stimulation response measurement (take the maximum value of each response). Similarly, $\Delta_{\mathrm{IPIP}}, \Delta_{\mathrm{IMCP}}$ (index finger), $\Delta_{\mathrm{MPIP}}, \Delta_{\mathrm{MMCP}}$ (middle finger); $\Delta_{\text {RPIP }}, \Delta_{\text {RMCP }}$ (ring finger); $\Delta_{\text {LMCP }}$ (little finger); $\Delta_{\text {EXT/FLEX }}$ (wrist extension/flexion); $\Delta_{\mathrm{ULN} / \mathrm{RAD}}$ (ulnar/radial deviation); $\Delta_{\mathrm{PRO} / \mathrm{SUP}}$ (pronation/supination) are the difference between maximum joint angles $\left(\varphi_{\mathrm{TMCP}}, \varphi_{\mathrm{IPIP}}, \varphi_{\mathrm{IMCP}}\right.$, $\varphi_{\mathrm{MPIP}}, \varphi_{\mathrm{MMCP}}, \varphi_{\mathrm{RPIP}}, \varphi_{\mathrm{RMCP}}, \varphi_{\mathrm{LMCP}}, \varphi_{\mathrm{EXT} / \mathrm{FLEX}}, \varphi_{\mathrm{ULN} / \mathrm{RAD}}$, and $\left.\varphi_{\text {PRO/SUP }}\right)$ measured in stimulation-generated and those measured in voluntary contraction. Finally, the offline analysis is used to select the best stimulation site for each target action with the smallest aggregate difference error.

2) On-line algorithm. The criterion requires high computational and time-consuming costs. Therefore, a simplified algorithm is utilized to realize real-time search of the optimal stimulation electrode. The on-line criterion is simplified as follows:

$$
\left\{\begin{array}{l}
\Delta_{\text {Thumb }, i}=\sqrt{\left(\frac{\Delta_{\mathrm{TMCP}}}{\varphi_{\mathrm{TMCP}}}\right)^{2}}, \\
\Delta_{\mathrm{Index}, i}=\frac{1}{2} \sqrt{\left(\frac{\Delta_{\mathrm{IPIP}}}{\varphi_{\mathrm{IPIP}}}\right)^{2}+\left(\frac{\Delta_{\mathrm{IMCP}}}{\varphi_{\mathrm{IMCP}}}\right)^{2}}, \\
\Delta_{\mathrm{Middl}, i}=\frac{1}{2} \sqrt{\left(\frac{\Delta_{\mathrm{MPIP}}}{\varphi_{\mathrm{MPIP}}}\right)^{2}+\left(\frac{\Delta_{\mathrm{MMCP}}}{\varphi_{\mathrm{MMCP}}}\right)^{2}}, \\
\Delta_{\mathrm{Ring}, i}=\frac{1}{2} \sqrt{\left(\frac{\Delta_{\mathrm{RPIP}}}{\varphi_{\mathrm{RPIP}}}\right)^{2}+\left(\frac{\Delta_{\mathrm{RMCP}}}{\varphi_{\mathrm{RMCP}}}\right)^{2}}, \\
\Delta_{\mathrm{Little}, i}=\sqrt{\left(\frac{\Delta_{\mathrm{LMCP}}}{\varphi_{\mathrm{LMCP}}}\right)^{2}}, \\
\Delta_{\mathrm{WExt} / \mathrm{Flex}, i}=\sqrt{\left(\frac{\Delta_{\mathrm{EXT} / \mathrm{FLEX}}}{\varphi_{\mathrm{EXT} / \mathrm{FLEX}}}\right)^{2}+\left(\frac{\Delta_{\mathrm{ULN} / \mathrm{RAD}}}{\varphi_{\mathrm{ULN} / \mathrm{RAD}}}\right)^{2}+\left(\frac{\Delta_{\mathrm{PRO} / \mathrm{SUP}}}{\varphi_{\mathrm{PRO} / \mathrm{SUP}}}\right)^{2}} .
\end{array}\right.
$$

Note that most variables in (3) are similar with (1) and (2). Therefore, the determination of the optimal electrode for the wrist extension, wrist flexion, five-finger extension and flexion were based on these six aggregate difference errors which considers only maximum joint excursions measured in dynamic conditions. The difference errors are calculated on-line for each pad individually and the procedure was applied for the dorsal and volar aspect electrodes independently.

\subsubsection{Android APP Design in Control Terminal}

An Android-based smartphone APP program is also developed to run the online algorithm. The APP is divided into a user interface (UI) thread and three child threads running network communication. The UI thread is respon- sible for providing an operating interface for users (illustrated in Fig. 3a). The UI interface mainly includes three sub-modules: the stimulator parameter setting module, which can set the stimulation parameters (pulse frequency, current intensity, stimulation on-time, and stimulation offtime); the multi-site electrode parameter setting module for setting the duration of each site stimulation; and the site optimization result display module, which is to provide the user with the optimal electrode. The flow chart of on-line determination of the optimal electrode is presented in Fig. 3b. When the user finishes setting the parameters in the UI and clicks the "Start Scanning" button (Fig. 3b), the above three child threads establish a TCP Socket connection with each of the three servers (the stimulator, electrode driving module and data glove). The UI thread will combine the setting parameters into a message string according to the instruction format and send that message to the three child threads. Then the motion data are read from data glove and six difference errors are calculated on-line for each pad individually. If greater than the last data stored, the aggregate difference error and stimulation site number will be updated. Then, the next round of site stimulation is performed, and the cycle continues until completion of the 20 -site polling stimulation. Finally, the optimal electrode number can be displayed in the UI interface.

\subsection{Intervention}

\subsubsection{Searching Optimal Stimulating Electrode Test}

Step 1: Instrumentation initialization and setup. The multi-pad electrode was positioned and attached to the volar surface of the forearm. The healthy subject was instructed to wear the wireless data glove and the EdisonSTIM stimulator, and the wireless electrode driving module was then connected to the stimulator. The whole prototype was initialized and tested to verify the wireless communication of data between each module.

Step 2: Online and real-time searching test. After all the checks were completed, the Android APP was opened in the control terminal, and the "Start Scanning" button was clicked (Fig. 3b). The following parameters were set for the Edison stimulator within the Android APP: frequency $20 \mathrm{~Hz}$, pulse width $250 \mu \mathrm{s}$, and intensity $20 \mathrm{~mA}$, with asymmetrical charge balanced pulses. All 20 sites in the multi-pad electrode were individually sequentially activated. The activation sequence was set for $2 \mathrm{~s}$ on, followed by $1 \mathrm{~s}$ off. Each site was repeated 2 times. The total duration of one trial of the recording session lasted for approximately $120 \mathrm{~s}$, and the actual stimulation time was $40 \mathrm{~s}$. In addition, the common motion analysis systems often sample at rates of $60 \mathrm{~Hz}$ to $120 \mathrm{~Hz}$ [13-15]. Therefore, considering the Nyquist rate and stimulation response undergo the modulation by the muscle reaction, the sample rate was set to $100 \mathrm{~Hz}$ to ensure the accuracy and stability of motion data collection and wireless transmission in the wireless Edison data glove. Finally, following the completion of the polling stimulation, the optimal electrode numbers were presented in the APP. The offline analysis based on the 
traditional criterion [17] (Section 2.2.2) was performed for the verification accuracy of the searching optimal stimulating electrode test.

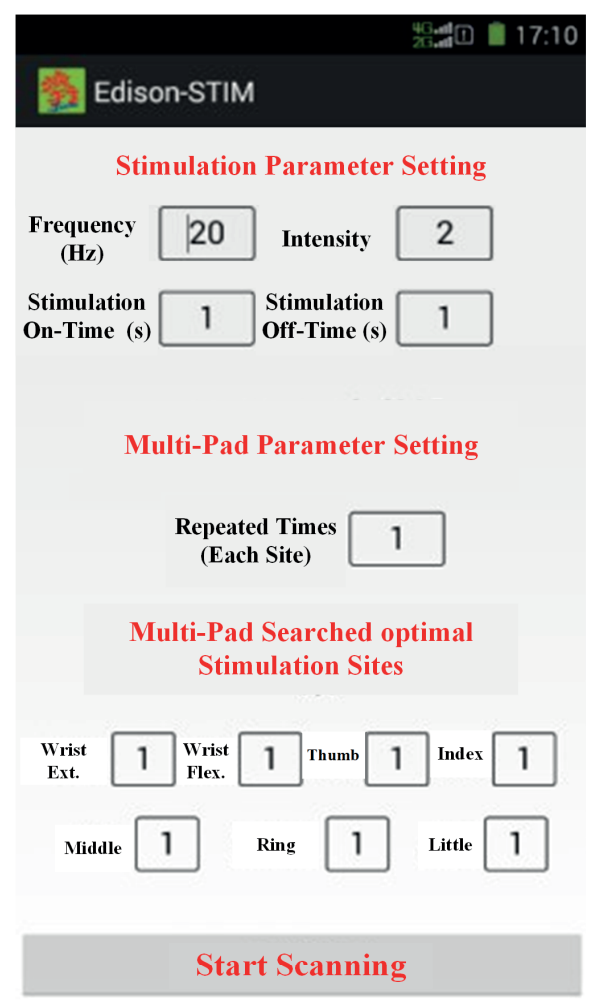

(a)

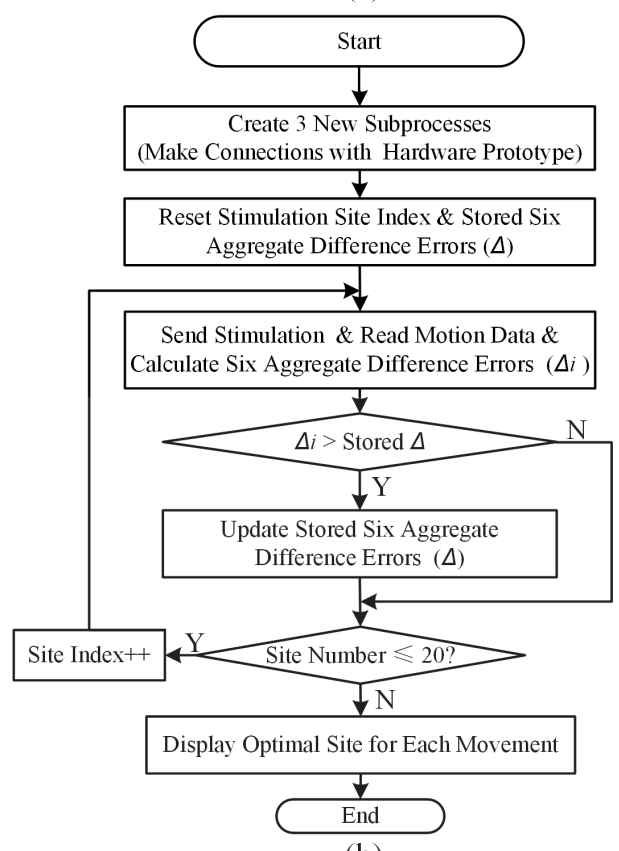

(b)

Fig. 3. (a) Android APP interface running on the control terminal Android smartphone. (b) Flow chart of the online stimulation electrode searching algorithm of targeted movement. *Notes: The stimulation on-time is the duration of a complete stimulation pulse sequence, while the stimulation off-time is the intervals of the two stimulation pulse sequences.

\subsubsection{Stimulation Muscle Fatigue Reduction Verification Test}

The test was divided into two groups. The subject's target muscles were stimulated by sending a stimulation pulse to the four active sites of the multi-pad electrode one by one with a $90^{\circ}$ phase shift between successive sites (Multi-site group). For better comparison, conventional single electrode stimulation (SES) was introduced as the control group (SES group). The four active sites of the multi-pad electrode with simultaneous stimulation noted above were considered one electrode. Therefore, in the SES group, the four sites of the multi-pad electrode were used to simulate the single electrode stimulation effect. For both groups, the resultant frequency delivered to the muscle was $40 \mathrm{~Hz}$ and the pulse width was $500 \mu \mathrm{s}$, with asymmetrical charge balanced pulses. The isometric wrist joint torque was measured during fatiguing stimulations lasting $80 \mathrm{~s}$. Each mode of stimulation was delivered a total of three times for each target muscle, and each test interval was required to last for at least half an hour. In addition, the selected four sites positions were "\#3", “\#4", “\#8" and "\#9" (refer to Fig. 2a). Therefore, in the Multi-site group, the alternating stimulation occurred in the order of "3-4-98" with a $10 \mathrm{~Hz}$ frequency at each site. The subject wearing the multi-site FES prototype for the fatigue test is presented in Fig. 4.

The subject was instructed to sit upright with the system worn on the upper arm, and a Velcro strap attached to the arm brace was used for wrist stabilization. The main target muscles in this test were the extensor carpi ulnaris (ECU) muscle and the flexor carpi radialis (FCR) muscle. A reference gelled stimulation electrode $\left(4 \times 4 \mathrm{~cm}^{2}\right)$ was placed $2 \mathrm{~cm}$ away from the multi-pad electrode near the elbow olecranon. The isometric wrist joint torque was measured using a custom-made device [18]. The torque signals were sampled with a 16-bit ADC acquisition board PCI-6220 (National Instruments Inc., USA) at a frequency of $1 \mathrm{kHz}$. A self-designed software was used to record the torque data on the PC for offline analysis. In order to avoid

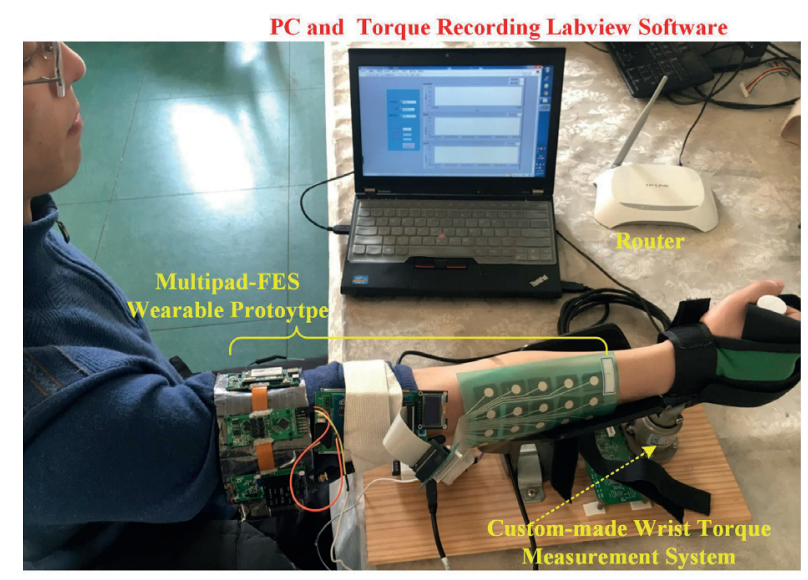

Fig. 4. Photograph of the subject wearing the multi-site FES prototype for fatigue test. 
the safety issue related to the induced current density and to comply with the standard [35], some measures and precautions should to be taken before the application of fatigue test on the subject. At first, the subject was required to carry out three maximal voluntary contractions (MVCs) in wrist flexion and extension with verbal prompts. Thus in each wrist flexion/extension repetition, the current which can elicit 30\% MVC was set as the stimulation intensity used in the fatigue stimulation. Simultaneously, the current density in the stimulation electrode should not exceed the safety level (maximum is $10 \mathrm{~mA} / \mathrm{cm}^{2}$ ). According to the procedures described above, the current intensity for the subject's wrist extension stimulation was set at $22 \mathrm{~mA}$, and the wrist flexion stimulation was set at $16 \mathrm{~mA}$. Secondly, a rest period of 5 hours was executed between fatigue tests to guarantee complete muscle force recovery. In addition, the subject should stop the experiment immediately when he experienced the skin pain or redness during the stimulation test. Finally, the fatigue stimulation tests were performed in the ECU and FCR muscles using the two protocols.

\subsection{Assessment}

\subsubsection{Searching Optimal Stimulating Electrode Test}

The searching optimal stimulating electrode test was performed twice by placing the multi-pad electrode on the dorsal and volar surfaces of the forearm. The metric Location Error ( $L E)$ was defined in the following equation:

$$
L E=\mid \text { ElectrodePosition }_{\text {online }}-\text { ElectrodePosition }\left._{\text {offline }}\right|_{\text {distance }} .
$$

It represented the distance between the positions of two electrodes selected by online test (ElectrodePosition online$_{\text {) }}$ and the offline analysis (ElectrodePosition offline$_{\text {e. }}$. According to the multi-pad electrode site distribution map illustrated in Fig. 2a, the axial and transversal interpad distances of two neighboring sites were $2.5 \mathrm{~cm}$ and $2 \mathrm{~cm}$. Through comparison with the offline analysis based on the aggregate difference [17], the accuracy of the searching selective stimulation could be indicated to some extent.

\subsubsection{Stimulation Muscle Fatigue Reduction Verification Test}

The following three metrics were used to evaluate two groups of stimulation muscle fatigue reduction [19]: (1) Fatigue index (FI) was defined as the torque at the end of the 80 s stimulation normalized to the maximum torque, which occurred near the beginning of the stimulation. (2) Fatigue time (FT) was defined as the time difference between the time at which the maximum torque was achieved and when the torque dropped to $3 \mathrm{~dB}$. (3) Torquetime integral (TTI) was defined as the integral of torque over the whole 80 s fatiguing stimulation.

To investigate the stimulation fatigue reduction performance, the paired t-test was implemented on three results $(F I, F T$, and $T T I)$. Statistical analysis was performed using SPSS statistics 19.0 software (IBM corp., Chicago, USA), and the results are reported as means \pm standard deviations (SDs). Differences with $P \leq 0.05$ were considered significant.

\section{Results}

\subsection{Online Test to Search for the Optimal Stimulating Electrode}

Figure 5a shows the photograph of the real-time online test for stimulation site optimization in the FCR muscle of one healthy subject using the multi-site FES system. The enlarged Android APP interface of the control terminal (Huawei C8813Q Android phone) is illustrated in Fig. 5b. It shows the results of the last automatic optimization site in the FCR muscles. The numbers in the APP interface are corresponding to the labels in Fig. 2a. Therefore, according to the online test, the optimal electrode position for the wrist flexion is the site "\#10" in the multipad electrode. Similarly, the optimal electrode positions of five fingers flexion are "\#3" (thumb), “\#6" (index), “\#6" (middle), "\#7” (ring) and "\#6" (little).

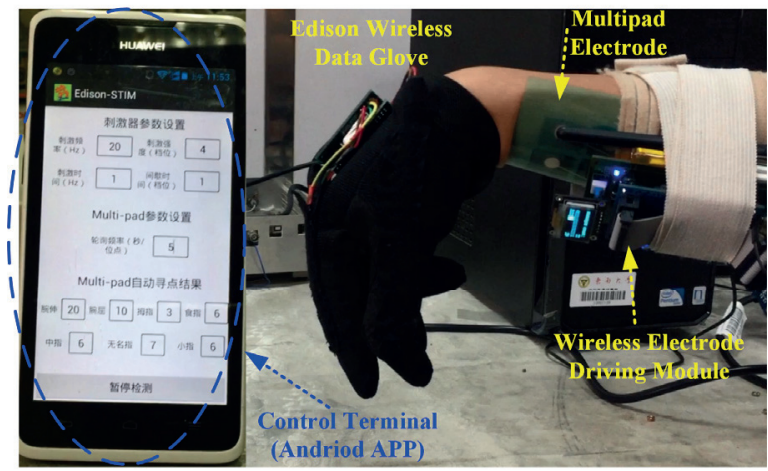

(a)

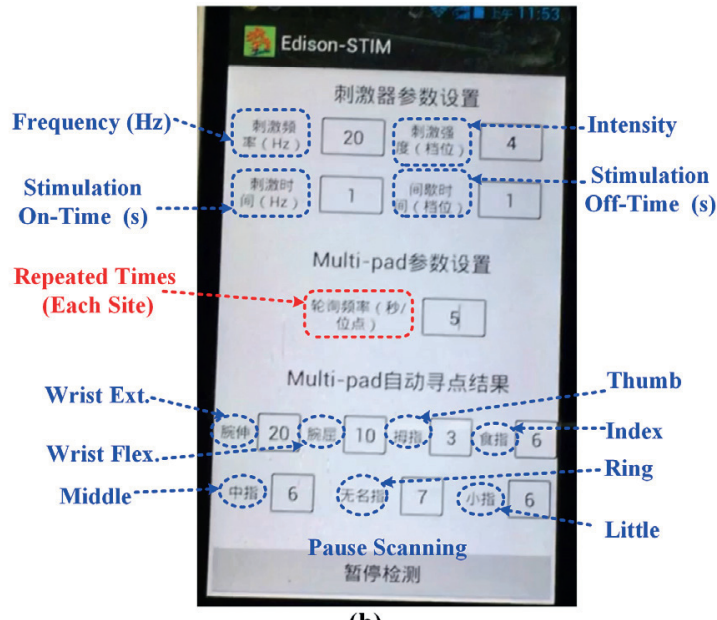

(b)

Fig. 5. (a) The photograph of the real-time online test for stimulation site optimization in the FCR muscle of one healthy subject using the multi-site FES system. (b) The enlarger photograph of control terminal in (a). 


\begin{tabular}{|c|c|c|c|}
\hline Movement & $\begin{array}{c}\text { Online } \\
\text { Electrode } \\
\text { Position }\end{array}$ & $\begin{array}{c}\text { Offline } \\
\text { Electrode } \\
\text { Position }\end{array}$ & $\begin{array}{c}\text { Location } \\
\text { Error } \\
\text { (cm) }\end{array}$ \\
\hline Wrist Ext. & $\# 2$ & $\# 2, \# 12$ & 0 \\
\hline Thumb Ext. & $\# 4$ & $\# 4$ & 0 \\
\hline Index Ext. & $\# 3$ & $\# 4$ & 2.5 \\
\hline Middle Ext. & $\# 9$ & $\# 14$ & 2.0 \\
\hline Ring Ext. & $\# 14$ & $\# 14$ & 0 \\
\hline Little Ext. & $\# 15$ & $\# 14$ & 2.5 \\
\hline Wrist Flex. & $\# 10$ & $\# 3, \# 14$ & 3.2 \\
\hline Thumb Flex. & $\# 3$ & $\# 3$ & 0 \\
\hline Index Flex. & $\# 6$ & $\# 2$ & 3.2 \\
\hline Middle Flex. & $\# 6$ & $\# 2$ & 3.2 \\
\hline Ring Flex. & $\# 7$ & $\# 2$ & 2.0 \\
\hline Little Flex. & $\# 6$ & $\# 2$ & 3.2 \\
\hline Average Ext. & -- & -- & 1.2 \\
\hline Average Flex. & -- & -- & 2.5 \\
\hline
\end{tabular}

Tab. 1. Optimal electrode positions for the corresponding movements obtained from the online test and the offline analysis. *Notes: the "\#number" is corresponding to the site labels of the electrode site distribution map in Fig. 2a.

Table 1 shows the optimal electrode positions of the corresponding movements obtained from the online test and the offline analysis. According to (1) and the electrode site distribution map in Fig. 2(a), the Location Error of each movement was calculated, and the average errors for the extension and flexion motions were $1.2 \mathrm{~cm}$ and $2.5 \mathrm{~cm}$, respectively. The online test demonstrated that the proposed multi-site FES prototype had acceptable accuracy in the automatic search for an optimal stimulation site.

\subsection{Fatigue Test Based on Multi-site FES}

Figure 6 shows the wrist extension and flexion torques produced during one 80 -s fatiguing stimulation trial in the Multi-site and SES groups. Although efforts were made to match the initial maximum torques produced across trials, the mean value for the Multi-site group was higher than that for the SES group. After reaching the maximum value, the torques decreased rapidly in the two modes due to the immediate generation of fatigue. However, using the multi-pad electrode with the distributed sequential stimulation method resulted in a clear decrease in fatigue compared with the conventional SES due to the lower torque declination rate during the first quarter of the stimulation and the higher wrist torque throughout the stimulation.

Figure 7 presents the means and SDs of the stimulation fatigue assessing metrics (FI, FT and TTI) for each trial of the two stimulation protocols after three trials. The mean FI value in the wrist extension stimulation was $0.415 \pm 0.057$ for the SES protocol and $0.603 \pm 0.046$ for the Multi-site protocol (Fig. 7a). The corresponding average $F T$ values were $(18 \pm 7) \mathrm{s}$ and $(54 \pm 10) \mathrm{s}$ (Fig. $7 \mathrm{~b}$ ), respectively, and the corresponding average TTI values were $(72.751 \pm 8.590) \mathrm{Nm} \cdot \mathrm{s}$ and $(80.625 \pm 1.675) \mathrm{Nm} \cdot \mathrm{s}$, respectively (Fig. 7c). For the wrist flexion stimulation, however, the mean $F I$ value was $(0.334 \pm 0.115)$ for the SES protocol and $(0.643 \pm 0.145)$ for the Multi-site protocol (Fig. 7d). The corresponding average $F T$ values were $(38 \pm 3) \mathrm{s}$ and $(65 \pm 14) \mathrm{s}$ (Fig. 7e), respectively, and the cor- responding average $T T I$ values were $(114.897 \pm 10.49) \mathrm{Nm} \cdot \mathrm{s}$ and (133.779 \pm 6.181$) \mathrm{Nm} \cdot \mathrm{s}$, respectively (Fig. 7f). The Multi-site group showed a $193 \%$ higher FI value for the wrist flexion stimulation $(t=17.655, P=0.003)$ (Fig. 7d) than for the SES protocol stimulation.

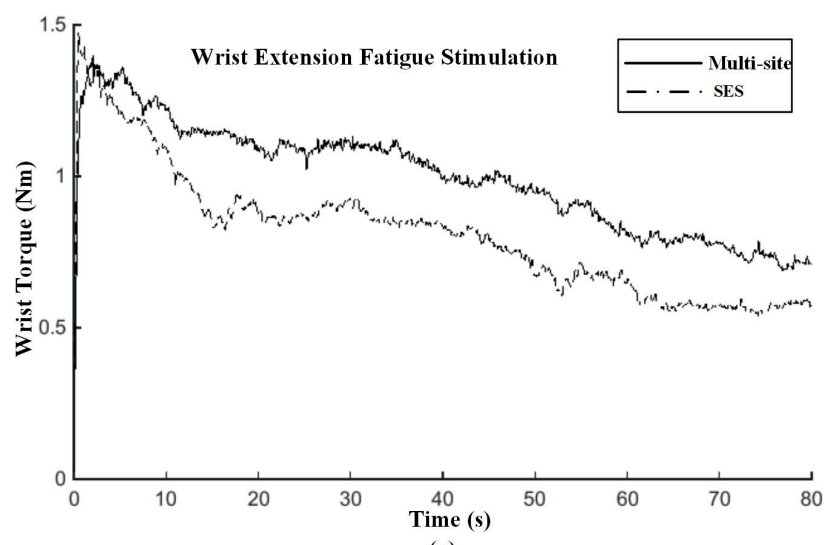

(a)

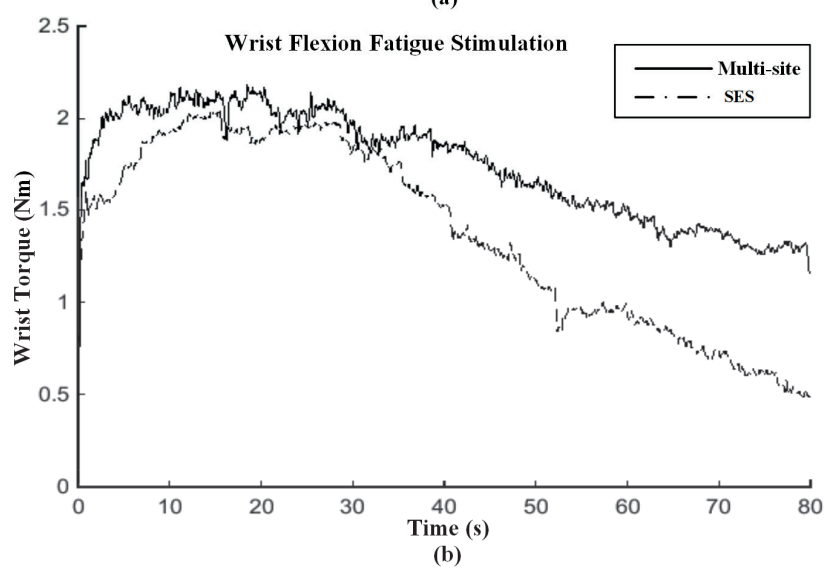

Fig. 6. Wrist torques produced during one 80 s fatiguing stimulation trial in the Multi-pad and SES groups. (a) Wrist extension torque under the fatigue stimulation. (b) Wrist flexion torque under the fatigue stimulation.
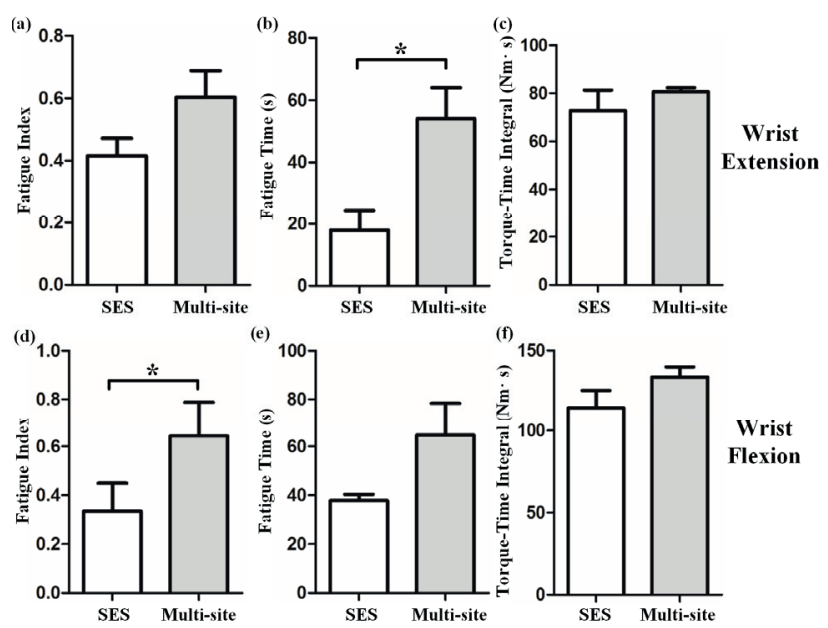

Fig. 7. Comparison of the fatigue assessment metrics for the SES and Multi-pad groups: (a) FI; (b) FT; and (c) TTI in the wrist extension fatigue stimulation. (d) FI; (e) FT; and (f) TTI in the wrist flexion fatigue stimulation. The data are presented as means of three trials, and error bars indicate one SD. All the differences were statistically significant $(P<0.001)$. 
Simultaneously, the FT results for the Multi-site protocol showed a significantly higher positive offset, with the fatigue time increasing to $300 \%$ from the SES group in the wrist extension $(t=-14, P=0.005)$ (Fig. 7b), indicating that the Multi-site protocol can produce more efficient muscle contraction over a much longer period of time. The above statistical test analysis results indicated that the multi-site protocol stimulation allows the muscle to maintain a high level of contraction until the end of the stimulation. Therefore, we can conclude that the use of the multipad electrode with alternating stimulation may result in a better reduction in stimulation fatigue.

\section{Discussion}

D. B. Popović et al. proposed the criterion for searching for the optimal site at which stimulation generates joint excursions that are minimally different from the joint excursions of the fingers, wrist and forearm measured in healthy individuals [17]. Because the selective stimulation site has a close relationship with the current density traveling through it into the tissue under the target site, studies have demonstrated that current density is highly related to the shape, spacing distance, and size of the electrode and the impedance of the stimulating electrode gel [7, 20, 21]. Therefore, the use of a multi-pad electrode is of great clinical value for determining the optimal site while considering changes in positions and shapes. In this paper, the optimal electrode searching offline method reported in [17], which is based on the aggregate error between the stimulationgenerated response and the voluntary motion response, was adopted in a PC to assess the on-line automatic search for an optimal stimulation site test. Because the offline analysis is not practical for use in clinical rehabilitation, we designed an APP based on an Android smartphone to determine the optimal stimulation site positioning with a simple algorithm. Although the online optimization algorithm described above can be determined in real-time, only a less accurate result can be provided for the determination of the stimulation site, and interference from other actions on the target motion is not considered when searching for the site. For example, when grasping, there will usually be a certain wrist flexion action, or the middle finger will also flex when the index and ring fingers are flexed. Therefore, the accuracy and performance of this real-time prototype needs to be improved with a more complex and robust algorithm in future studies. The electrode (including size, material, and stimulation parameters), interference of non-agonist muscles, rapid muscle fatigue, instrumental errors, individual differences in forearm anatomy (muscle overlap) and the tissue characteristics (large dissipation of the induced charge) will also be considered in the future.

In addition, there are some references using the simultaneous activation of two or more electrodes. For example, three [11] or eight [17] selected pads are simultaneously activated to yield better performance. However, in the electrode searching phase, the pads in the multi-pad electrode are all activated in turn (sequentially one by one).
Only [10] has described that stimulation via electrode pads placed at locations selected according to significant differences in the EMG maps obtained from the paretic and nonparetic forearms. As far as authors know, there is no reference investigating the employing simultaneous activation of two or more electrodes in the searching phase, and the influence to the stimulation electrode determination has yet to be studied

Healthy muscle is composed of normal type I (S type) and IIa fibres (fatigue-resistant MU dominating, FR type) and type IIb fibres (fast-fatigable MUs dominating, FF type). During voluntary contractions, the CNS controls muscle force modulated by both the number of activated MUs (recruitment coding) and the activation frequency (rate coding) [22], [23]. The voluntary recruitment order of MUs follows Henneman's size principle; the CNS recruits the three types of MUs in the order of S-FR-FF according to the frequency of the muscle force required by the task from small to large and the activation frequency and therefore asynchronously completes the cooperation [22]. Therefore, the CNS starts with small MUs that innervate fatigue-resistant MUs and continues with large MUs that innervate fast-fatigable MUs [25], [26]. In contrast, although the order in which MUs are recruited during FES remains unclear, it is generally agreed to occur in a nonphysiological reverse recruitment order [27-30], with type II fibers recruited first, while the smaller type I muscle fibers require further increases in the stimulation intensity for activation. In addition, in electrical stimulation, all types of MUs in the muscle are synchronously activated to rapidly contract, replacing the activation frequency and firing rate of the MUs under normal physiological activities [31]. Therefore, use of FES results in a higher energy demand and faster onset of muscle fatigue [32]. Furthermore, in SCI subjects, the paralyzed muscle loses normal type I and IIa fibers and becomes predominantly composed of type IIb fibers, thus increasing muscle fatigue [33]. Therefore, it is difficult to solve the reverse problem of FES using existing methods, and some researchers have proposed the adoption of asynchronous stimulation to alleviate muscle fatigue [19], [34]. For a synergistic muscle, the different MUs are asynchronously stimulated at different frequencies or pulse widths for the same stimulation duration, and stimulation of each site produces a resultant force that can maintain a certain level of convergence and contraction. Compared to the single-synchronous stimulation, the activation frequency of each MU is reduced, which can reduce muscle fatigue. Nguyen et al. [19] introduced the spatially distributed sequential stimulation (SDSS) method with four-site stimulation electrodes to effectively reduce stimulation muscle fatigue. Compared with previous experiments, we used a self-designed multi-pad stimulation electrode and corresponding control systems to achieve simultaneous single-electrode stimulation with multi-pad electrodes, thus achieving SDSS and single-electrode stimulation. The same stimulation intensity and stimulating electrode area in the fatigue test can be guaranteed, and while previous studies have mainly focused on lower limb muscles, this study was the first to examine the stimulation 
fatigue of the upper wrist extensors and wrist flexors. According to the fatigue test, the obtained results were consistent with previous studies [19], so it can therefore validate the fatigue reduction performance of the multi-site FES prototype.

Furthermore, literature [21] has showed that the stimulation spatial current densities through into the target tissues are highly related with the electrode sizes, shapes and spacing distance. Larger electrodes can avoid the risk of high current densities, but it remains a challenge to reduce the stimulation selectivity. In contrast, the smaller electrodes can enhance the selectivity with the cost of higher current densities. According to the reviewed references, the typical electrode area is $0.5 \mathrm{~cm}^{2}$ (circular shape, $D=8 \mathrm{~mm}$ ) [10], $0.8 \mathrm{~cm}^{2}$ (circular shape, $D=10 \mathrm{~mm}$ ) [17], and $2.52 \mathrm{~cm}^{2}$ (rectangular shape, $1.4 \mathrm{~cm} \times 1.8 \mathrm{~cm}$ ). Therefore, the trade-off between the stimulation selectivity and current density need to be further studied in our future research.

\section{Conclusion}

This case study shows the feasibility of improving selective stimulation and reducing stimulation muscle fatigue in upper extremity rehabilitation using a selfdesigned multi-site FES prototype. The results of this study suggest that the proposed multi-site FES prototype may have positive effects in healthy subjects. The small sample size of this study does not prove the strength of the association between using the multi-site FES prototype and improving selective stimulation and reducing stimulation muscle fatigue. Therefore, further investigation of multisite FES in patients with stroke or SCI is warranted.

\section{Acknowledgments}

This work was supported by the National Natural Science Foundation of China (61801262, 61534003, 81701806, and 61874024), the Natural Science Foundation of the Jiangsu Higher Education Institutions of China (18KJB510039), the Science \& Technology Pillar Program of Jiangsu Province (BE2016738), and the Fundamental Research Funds for the Central Universities (2242020R20010).

\section{References}

[1] POPOVIC-MANESKI, L., KOSTIC, M., BEJELIC, G., et al. Multi-pad electrode for effective grasping: Design. IEEE Transactions on Neural Systems and Rehabilitation Engineering, 2013, vol. 21, no. 4, p. 648-654. DOI: 10.1109/TNSRE.2013.2239662

[2] LONG, C. An electrophysiologic splint for the hand. Archives of Physical Medicine and Rehabilitation, 1963, vol. 44, p. 499-503. PMID: 14050723
[3] MERLETTI, R., ACIMOVIC, R., GROBELNIK, S., CVILAK, G. Electrophysiological orthosis for the upper extremity in hemiplegia: feasibility study. Archives of Physical Medicine and Rehabilitation, 1975, vol. 56, no.12, p. 507-513. PMID: 1081869

[4] MALESEVIC, N. M., POPOVIC-MANESKI, L. Z., ILIC, V., et al. A multi-pad electrode based functional electrical stimulation system for restoration of grasp. Journal of Neuroengineering and Rehabilitation, 2012, vol. 9, p. 1-12. DOI:10.1186/1743-0003-9-66

[5] NATHAN, R. H., OHRY, A. Upper limb functions regained in quadriplegia: a hybrid computerized neuromuscular stimulation system. Archives of Physical Medicine and Rehabilitation, 1990, vol. 71, no.6, p. 415-421. PMID: 2334287

[6] FUJII, T., SEKI, K., HANDA, Y. Development of a new FES system with trained super-multichannel surface electrodes. In Proceedings of 9th Annual Conference IFESS. Bournemouth (UK), 2004, p. 21-24.

[7] KELlER, T., LAWRENCE, M., KUHN, A., et al. New multichannel transcutaneous electrical stimulation technology for rehabilitation. In 2006 International Conference of the IEEE Engineering in Medicine and Biology Society (EMBS 2006). New York (USA) 2006, p. 194-197. DOI: 10.1109/IEMBS.2006.259399

[8] POPOVIC-BIJELIC, A., BIJELIC, G., JORGOVANOVIC, N., et al. Multi-field surface electrode for selective electrical stimulation. Artificial Organs, 2005, vol. 29, p. 448-452. DOI: 10.1111/j.15251594.2005.29075.x

[9] O’DWYER, S., O’KEEFFE, D., COOTE, S., et al. An electrode configuration technique using an electrode matrix arrangement for FES-based upper arm rehabilitation systems. Medical Engineering \& Physics, 2006, vol. 28, no. 2, p. 166-176. DOI: 10.1016/j.medengphy.2005.03.010

[10] POPOVIC-MANESKI, L., TOPALOVIC, I., JOVICIC, N., et al. Stimulation map for control of functional grasp based on multichannel EMG recordings. Medical Engineering \& Physics, 2016, vol. 38, no. 11, p. 1251-1259. DOI: 10.1016 j.medengphy.2016.06.004

[11] CREMA, A., MALESEVIC, N., FURFARO, I., et al. A wearable multi-site system for NMES-based hand function restoration. IEEE Transactions on Neural Systems and Rehabilitation Engineering, 2018, vol. 26, no. 2, p. 428-440. DOI: 10.1109/TNSRE.2017.2703151

[12] JAHANSHAHI, J. A., DANYALI, H., HELFROUSH, M. S. A modified compressed sensing-based recovery algorithm for wireless sensor networks. Radioengineering, 2019, vol. 28, no. 3, p. 610-617. DOI: $10.13164 /$ re.2019.0610

[13] SIMONE, L. K., SUNDARRAJAN, N., LUO, X., et al. A low cost instrumented glove for extended monitoring and functional hand assessment. Journal of Neuroscience Methods, 2007, vol. 160, no. 2, p. 335-348. DOI: 10.1016/j.jneumeth.2006.09.021

[14] OESS, N. P., WANEK, J., CURT, A. Design and evaluation of a low-cost instrumented glove for hand function assessment. Journal of Neuroengineering and Rehabilitation, 2012, vol. 9, p. 1-11. DOI: $10.1186 / 1743-0003-9-2$

[15] GENTNER, R., CLASSEN, J. Development and evaluation of a low-cost sensor glove for assessment of human finger movements in neurophysiological settings. Journal of Neuroscience Methods, 2009, vol. 178, p. 138-147. DOI: 10.1016/j.jneumeth.2008.11.005

[16] WANG, H. P., GUO, A. W, ZHOU, Y. X., et al. A wireless wearable surface functional electrical stimulator. International Journal of Electronics, 2017, vol. 104, no. 9, p. 1514-1526. DOI: $10.1080 / 00207217.2017 .1312708$

[17] POPOVIC, D. B., POPOVIC, M. B. Automatic determination of the optimal shape of a surface electrode: selective stimulation. Journal of Neuroscience Methods, 2009, vol. 178, p. 174-181. DOI: $10.1016 /$ j.jneumeth.2008.12.003 
[18] ZHOU, Y. X., WANG, H. P., BAO, X. L, et al. A frequency and pulse-width co-modulation strategy for transcutaneous neuromuscular electrical stimulation based on sEMG time-domain features. Journal of Neural Engineering, 2015, vol. 13, p. 1-15. DOI: $10.1088 / 1741-2560 / 13 / 1 / 016004$

[19] NGUYEN, R., MASANI, K., MICERA, S., et al. Spatially distributed sequential stimulation reduces fatigue in paralyzed triceps surae muscles: a case study. Artificial Organs, 2011, vol. 35, p. 1174-1180. DOI: 10.1111/j.1525-1594.2010.01195.x

[20] KUHN, A., KELlER, T., MICERA, S., et al. Array electrode design for transcutaneous electrical stimulation: A simulation study. Medical Engineering \& Physics, 2009, vol. 31, p. 945-951. DOI: 10.1016/j.medengphy.2009.05.006

[21] KUHN, A., KELLER, T., LAWRENCE, M., et al. The influence of electrode size on selectivity and comfort in transcutaneous electrical stimulation of the forearm. IEEE Transactions on Neural Systems and Rehabilitation Engineering, 2010, vol. 18, no. 3, p. 255-262. DOI: 10.1109/TNSRE.2009.2039807

[22] DE LUCA, C., LE FEVER, R., MCCUE, M., et al. Behaviour of human motor units in different muscles during linearly varying contractions. The Journal of Physiology, 1982, vol. 329, no. 1, p. 113-128. DOI: 10.1113/jphysiol.1982.sp014293

[23] KAMEN, G., DU, D. C. C. Independence of motor unit recruitment and rate modulation during precision force control. Neuroscience, 1999, vol. 88, no. 2, p. 643-653. DOI: 10.1016/S0306-4522(98)00248-6

[24] KRALJ, A. R., BAJD, T. Functional Electrical Stimulation: Standing and Walking after Spinal Cord Injury. CRC Press, 1989. ISBN 9780849345296

[25] HENNEMAN, E. Relation between size of neurons and their susceptibility to discharge. Science, 1957, vol. 126, no. 3287 , p. 1345-1347. DOI: $10.1126 /$ science. 126.3287 .1345

[26] JABRE, J. F., SPELLMAN, N. T. The demonstration of the size principle in humans using macro electromyography and precision decomposition. Muscle \& Nerve, 1996, vol. 19, no. 3, p. 338-341. DOI: 10.1002/(SICI)1097-4598(199603)19:3<338::AID-MUS9> 3.0.CO;2-E

[27] PECKHAM, P., MORTIMER, J., MARSOLAIS, E. Alteration in the force and fatigability of skeletal muscle in quadriplegic humans following exercise induced by chronic electrical stimulation. Clinical Orthopaedics and Related Research, 1976, vol. 114, p. 326-334. DOI: 10.1097/00003086-197601000-00041

[28] MOHR, T., ANDERSEN, J. L., BIERING-SORENSEN, F., et al. Long term adaptation to electrically induced cycle training in severe spinal cord injured individuals. Spinal Cord, 1997, vol. 35, p. 1-16. DOI: $10.1038 /$ sj.sc. 3100343

[29] PECKHAM, P. H. KNUSTSON, J. S. Functional electrical stimulation for neuromuscular applications. Annual Review of Biomedical Engineering, 2005, vol. 7, p. 327-360. DOI: 10.1146/annurev.bioeng. 6.040803.140103

[30] SHEFFLER, L. R., CHAE, J. Neuromuscular electrical stimulation in neurorehabilitation. Muscle \& Nerve, 2007, vol. 35, no. 5, p. 562-590. DOI: $10.1002 /$ mus. 20758

[31] DE LUCA, C. J. Myoelectrical manifestations of localized muscular fatigue in humans. Critical Reviews in Biomedical Engineering, 1984, vol. 11, no. 4, p. 251-279. PMID: 6391814

[32] SCOTT BICKEL, C., GREGORY, C. M., DEAN, J. C. Motor unit recruitment during neuromuscular electrical stimulation: A critical appraisal. European Journal of Applied Physiology, 2011, vol. 111, p. 1-9. DOI: 10.1007/s00421-011-2128-4

[33] BIERING-SORENSEN, B., BRUUN KRISTENSEN, I., KJAER, M., et al. Muscle after spinal cord injury. Muscle \& Nerve, 2009, vol. 40, no. 4, p. 499-519. DOI: 10.1002/mus.21391
[34] MALESEVIC, N. M., POPOVIC, L. Z., SCHWIRTLICH, L., et al Distributed low-frequency functional electrical stimulation delays muscle fatigue compared to conventional stimulation. Muscle \& Nerve, 2010, vol. 42, no. 4, p. 556-562. DOI :10.1002/mus.21736

[35] IEC 62226-3-1:2007/AMD1:2016, Exposure to electric or magnetic fields in the low and intermediate frequency range Methods for calculating the current density and internal electric field induced in the human body, Part 3-1: Exposure to electric fields - Analytical and 2D numerical models. European Standard EN 62226-3-1, 2016, p. 1-54.

\section{About the Authors...}

Haipeng WANG was born in Shandong, China in 1987. He received his Ph.D. degree from Southeast University in 2017. His research interests include biomedical circuits and systems design and biomedical signal processing.

Zhengyang BI was born in Jiangsu, China in 1991. He received his M.S. degree in Biomedical Engineering from Southeast University in 2016. His research interests include biomedical signal processing.

Yuxuan ZHOU was born in Jiangsu, China in 1985. He received his Ph.D. degree from Southeast University, in 2016. His research interests include the biomedical signal processing.

Fei LI was born in Jiangsu, China in 1980. He received his $\mathrm{Ph} . \mathrm{D}$. degree from Tokyo Institute of Technology, in 2013. His research interest is mixed signal circuits, intelligent CMOS sensors and data readout circuits.

Keping WANG was born in Jiangsu, China in 1980. He received his Ph.D. degree from the Southeast University in 2010. His research interests include future wireless bioelectrical interfaces, body-area-networks, and implantable devices demand wireless technology.

Xiao-Ying LÜ (corresponding author) was born in Shanghai, China. She received her M.-Med. degree from Shanghai Second Medical University in 1986 and her Dr.-Dent. degree from Freiburg University, Freiburg, Germany, in 1996. In 1997, she became an associate professor of Southeast University, China and since 2003 she has been a fullprofessor.

Zhi-Gong WANG (corresponding author) was born in Henan, China. He received the M.-Eng. degree in Radio Engineering from Nanjing Institute of Technology (now, Southeast University), Nanjing, China, in 1981, and the Dr.-Ing. degree in Electronic Engineering from Ruhr-University Bochum, Germany, in 1990. Since October 1997, he has been a Full Professor with Southeast University, Nanjing, China. He has recently been involved with IC design for optic-fiber transmission systems, for RF wireless, microwave, and millimeter-wave applications, and in microelectronic systems for biomedical applications. 